\title{
Herbal Medicine (Sihogayonggolmoryeo-Tang or Chai-Hu-Jia-Long-Gu-Mu-Li-Tang) for Treating Hypertension: A Systematic Review and Meta-Analysis
}

\author{
Boram Lee $\mathbb{D}^{1}$ and Chan-Young Kwon $\mathbb{D}^{2}$ \\ ${ }^{1}$ Clinical Medicine Division, Korea Institute of Oriental Medicine, 1672 Yuseongdae-ro, Yuseong-gu, Daejeon 34054, \\ Republic of Korea \\ ${ }^{2}$ Department of Oriental Neuropsychiatry, Dong-eui University College of Korean Medicine, 62 Yangjeong-ro, Busanjin-gu, \\ Busan 47227, Republic of Korea
}

Correspondence should be addressed to Boram Lee; qhfka9357@naver.com

Received 14 July 2020; Accepted 27 August 2020; Published 9 September 2020

Academic Editor: Hong Chang

Copyright (c) 2020 Boram Lee and Chan-Young Kwon. This is an open access article distributed under the Creative Commons Attribution License, which permits unrestricted use, distribution, and reproduction in any medium, provided the original work is properly cited.

\begin{abstract}
Introduction. For situations in which effective and safe natural-derived products to treat hypertension are needed, recent studies suggest that an herbal medicine, Sihogayonggolmoryeo-tang (SYM), can improve both hypertension and concurrent mood symptoms. We aimed to evaluate the effectiveness and safety of SYM in treating hypertension. Methods. Thirteen English, Korean, and Chinese databases were comprehensively searched from their inception to May 2020. Randomized controlled trials (RCTs) using SYM as a monotherapy or adjunctive therapy for hypertension were evaluated. The primary outcome was the systolic and diastolic blood pressure (BP). Descriptive analyses of the relevant data were conducted, and where appropriate data were available, a meta-analysis was performed, and the results were presented as a risk ratio or mean difference with $95 \%$ confidence intervals. The risk of bias was assessed using the Cochrane risk of bias tool, and the quality of evidence was evaluated using the Grading of Recommendations, Assessment, Development and Evaluation (GRADE) approach. Results. Seven RCTs with 711 participants were included. Compared with placebo, SYM significantly lowered systolic and diastolic BP and concurrent depression. SYM significantly lowered systolic and diastolic BP compared with active controls; however, subgroup analysis revealed no differences between SYM and antihypertensives. In addition, SYM significantly decreased the level of concurrent depression compared with antidepressants. There was no consistent difference in BP reduction between SYM combined with antihypertensives and antihypertensives alone. No serious adverse events were reported following SYM administration. Most of the included studies had an unclear risk of bias, and the quality of evidence was generally rated "low." Conclusion. Current evidence suggests that SYM may have the potential to lower hypertension and concurrent depressive symptoms without serious adverse events. Additional highquality, placebo-controlled RCTs should be conducted to confirm the efficacy of SYM.
\end{abstract}

\section{Introduction}

Hypertension is a major public health challenge worldwide. It is strongly associated with potentially severe conditions including cardiovascular disease and premature death [1]. According to a comprehensive systematic analysis, the proportion of ischemic heart disease disability-adjusted life years attributable to high blood pressure (BP) was $53 \%$ in 2010 [2]. In the study, high BP was identified as the leading risk factor that affects the global disease burden, followed by tobacco smoking and alcohol use [2]. The prevalence of hypertension in adults worldwide in 2010 was estimated at $31.1 \%$ [3], but the proportion of patients treated and/or managed with proper medication is still low [4]. In addition, about 1 in 5 patients have apparent treatment-resistant hypertension, which is unresponsive to adequate antihypertensives [5]. More than 20\% of hypertension patients taking antihypertensives experience side effects such as 
impotence and emotional distress [6], which are the primary determinants of low adherence to antihypertensives [7]. Decreasing sodium intake, weight loss, increasing physical activity, decreasing alcohol intake, and eating a healthy diet including the Dietary Approaches to Stop Hypertension (DASH) diet are healthy lifestyle strategies to reduce the risk of hypertension [8]. However, the development of hypertension due to poor lifestyle habits such as sedentary behavior is still frequently reported [9]. In other words, hypertension remains a major public health issue, and conventional treatment and management strategies still need to be improved.

Some hypertensive patients are interested in complementary and alternative medicine (CAM) modalities. According to the 2012 National Health Interview Survey and the Adult Alternative Medicine supplement, a combination of hypercholesterolemia, hypertension, diabetes, and obesity as dyads and triads was significantly related to the increased use of CAM such as mind-body interventions, manipulative methods, and energy therapies [10]. It has been reported that some supplements/foods including coenzyme Q10, vitamin $\mathrm{D}$, and polyphenol-rich dark chocolate, and mind-body medicine including qigong, breathing, and meditation may be helpful for reducing BP [11]. Herbal medicine (HM) has been used as a treatment approach in East Asian traditional medicine (EATM) to treat various medical conditions including hypertension-related symptoms for thousands of years in East Asia. Today, HM is receiving much attention as an option to replace or supplement conventional medicines, and its effectiveness has already been demonstrated in some diseases including cardiovascular disease and hypertension [12-14].

Sihogayonggolmoryeo-tang (SYM) is a traditional HM used to treat insomnia, anxiety, irritability, and hypertension-related symptoms. In particular, since this HM is well-known for its mood-stabilizing effects [15-17], it may be helpful in improving not only hypertension but also concurrent mood symptoms. In addition, preclinical evidence suggests that SYM can exert a protective effect on the cardiovascular system by enhancing the function of endothelial progenitor cells, inducing antioxidant effects, and inhibiting vasoconstriction [18-20]. However, the efficacy of SYM in the treatment of hypertensive patients has not been systematically summarized and evaluated. Therefore, we aimed to synthesize the available evidence related to the effectiveness and safety of SYM as a monotherapy or adjunctive therapy for patients with hypertension and to assess the methodological quality of these studies to help clinicians establish evidence-based treatment strategies.

\section{Materials and Methods}

The protocol for this review was registered in the International Prospective Register of Systematic Reviews, PROSPERO (registration number: CRD42020187174). We reported this review in accordance with the Preferred Reporting Items for Systematic Reviews and Meta-Analyses statement [21]. Additionally, we followed the methods of Dr. Lee [22].
2.1. Data Sources and Search Strategy. One researcher (BL) comprehensively searched total 13 electronic databases on May 19, 2020: 5 English databases (Medline (via PubMed), EMBASE (via Elsevier), the Cochrane Central Register of Controlled Trials (CENTRAL), the Allied and Complementary Medicine Database (AMED) (via EBSCO), and the Cumulative Index to Nursing and Allied Health Literature (CINAHL) (via EBSCO)), five Korean databases (Oriental Medicine Advanced Searching Integrated System (OASIS), Korean studies Information Service System (KISS), Research Information Service System (RISS), Korean Medical Database (KMbase), and Korea Citation Index (KCI)), and three Chinese databases (China National Knowledge Infrastructure (CNKI), Wanfang data, and VIP). We also searched the reference lists of included studies and Google Scholar to identify additional eligible studies. Grey literature such as degree theses was also included. No language, publication date, and publication status restrictions were imposed. The following search terms were used in PubMed: Hypertension [MH] OR hypertens * [TIAB] OR "blood pressure" [MH] OR "blood pressure" [TIAB] OR blood pressure [TIAB] AND Chai-Hu-Jia-Long-Gu-Mu-Li-Tang [TIAB] OR Chai-Hu-Jia-Long-Gu-Mu-Li-Wan [TIAB] OR Chai-Hu-Jia-Long-Gu-Mu-Li-Pian [TIAB] OR Saiko-karyukotsu-borei-to [TIAB] OR Saiko-ka-ryukotsu-borei-to [TIAB] OR Sihogayonggolmoryeo-tang [TIAB] (Supplement 1).

\subsection{Inclusion Criteria}

2.2.1. Types of Studies. We included only randomized controlled trials (RCTs). We also included RCTs using the expression "randomization" without descriptions of the randomization methods. We excluded RCTs in which a quasirandom method was used for the allocation of treatments such as using alternate allocation or allocation by birth date.

2.2.2. Types of Participants. Studies on hypertension patients, diagnosed according to the international hypertension criteria, were included. There was no restriction on age, sex, race, or comorbidity of participants. We excluded trials that included patients suffering from other serious medical conditions such as cancer, liver disease, or kidney disease.

2.2.3. Types of Interventions. We included studies on SYM as a treatment intervention. HMs are known as "modified HMs" when the components are altered to achieve increased efficacy $[23,24]$. Thus, we also included studies in which modified forms of SYM, described as "modified SYM" and containing more than $50 \%$ of the components of the original prescription, were used as treatment interventions. Modified SYM in the included studies must contain Bupleuri Radix, Fossilia Ossis Mastodi, and Ostreae Testa, one of the most critical herbal components of SYM. For the dosage form, we considered only oral administration of SYM. Placebo, no treatment, and active controls such as conventional 
medication were included as control interventions. Studies involving SYM combined with other therapies as treatment interventions were also included if the other therapies were equally used in both the treatment and control groups. However, we excluded studies comparing different types of HM. There was no restriction on the treatment duration of SYM.

2.2.4. Types of Outcome Measures. The primary outcome was the systolic BP (SBP) and diastolic BP (DBP) measured after treatment. The secondary outcomes included (1) symptoms associated with hypertension such as insomnia and anxiety after treatment, (2) total effective rate (TER), calculated secondarily based on improvements in BP or other clinical symptoms, (3) the incidence of adverse events during the study period, and (4) quality of life after treatment.

2.3. Study Selection and Data Extraction. We removed duplicates in the search results obtained from the databases and additional sources and screened the titles and abstracts for eligibility using EndNote X8. Afterward, we evaluated the full texts of the eligible articles for final inclusion. For the included studies, we extracted information related to the study characteristics (author, publication year, country, and study design); approval from institutional review boards; informed consent; sample size and the number of dropouts; details about the participants, intervention, and comparisons; duration of the intervention and follow-up; outcome measures; results; and adverse events using a standardized data collection form (Excel 2016, Microsoft, Redmond, WA, USA). We also extracted the components, dosage form, and administration duration and frequency of SYM. The corresponding authors of the included studies were contacted via e-mail if there were insufficient data.

One researcher (BL) performed the abovementioned study selection and data extraction procedures, and another researcher (CYK) cross-checked the process. Any disagreement was resolved through discussion between the researchers.

2.4. Quality Assessment. We evaluated the risk of bias for the included RCTs using the Cochrane Collaboration's risk of bias tool [25]. The studies were classified as "low risk," "unclear," or "high risk" for each of the following domains: random sequence generation, allocation concealment, blinding of participants and personnel, blinding of outcome assessments, completeness of outcome data, selective reporting, and other potential bias. We evaluated the other potential bias domain with an emphasis on possible baseline imbalances between the treatment and control groups, such as the baseline BP level.

Using the Grading of Recommendations, Assessment, Development and Evaluation (GRADE) approach, we evaluated the quality of evidence for major findings with the online program GRADEpro (https://gradepro.org/) [26]. The risk of bias, inconsistency, indirectness, and imprecision of the results and the probability of publication bias were evaluated, and we classified the results into one of four groups: "very low," "low," "moderate," or "high."

One researcher (BL) conducted the abovementioned quality assessment, while another researcher (CYK) crosschecked the data. Any discrepancy was resolved through discussion between them.

2.5. Data Synthesis and Analysis. We conducted a narrative synthesis of the details of the participants, interventions, comparators, and outcomes for all included studies. In the case of primary or secondary outcome of this review, we quantitatively pooled the results using the Review Manager software, version 5.3 (Cochrane, London, UK). Continuous or dichotomous variables were pooled using mean differences (MD) or risk ratios (RRs), with 95\% confidence intervals (CIs). We evaluated heterogeneity between the studies included in each meta-analysis in terms of the effect measures using both the $\chi^{2}$ test and the $I^{2}$ statistic. $I^{2}$ values $\geq 50 \%$ and $\geq 75 \%$ were considered indicative of substantial and considerable heterogeneity. The meta-analyzed results were pooled using a random-effect model if the included studies had significant heterogeneity (an $I^{2}$ value $\geq 50 \%$ ). A fixed-effect model was used when the heterogeneity was not significant or the number of studies included in the metaanalysis was very small, which leads to poor precision for the estimate of between-study variance [27, 28]. Subgroup analysis was conducted according to the type of active controls used. Sensitivity analyses were planned to identify the robustness of meta-analysis results by excluding [1] studies with high risks of bias and [2] outliers. We also planned to assess the evidence of publication bias using a funnel plot if there were enough studies.

\section{Results}

3.1. Description of Studies. A total of 104 articles were identified from searching 13 databases, and no records were identified through other sources. After removing $37 \mathrm{du}-$ plicates, 49 articles were excluded based on screening the titles and abstracts. Through the full-text evaluation, 11 articles, specifically five case reports, one review article, two non-RCTs, two non-SYM-related articles, and one article comparing two different HMs, were excluded. Therefore, seven articles [29-35] with 711 participants were included in the qualitative and quantitative synthesis (Figure 1).

3.2. Characteristics of Studies. All studies were conducted in China. One article was a thesis [29], and the rest were journal articles. The included studies were as follows: one study comparing SYM with placebo [33], two studies comparing SYM with active controls [30, 35], and three studies comparing SYM plus active controls with active controls only $[29,32,34]$. One study was a three-arm parallel study comparing SYM, active controls, and SYM plus active controls [31]. For the active controls, antihypertensives including captopril, amlodipine besylate, and benazepril were used in four studies $[29,31,32,34]$ and antidepressants 


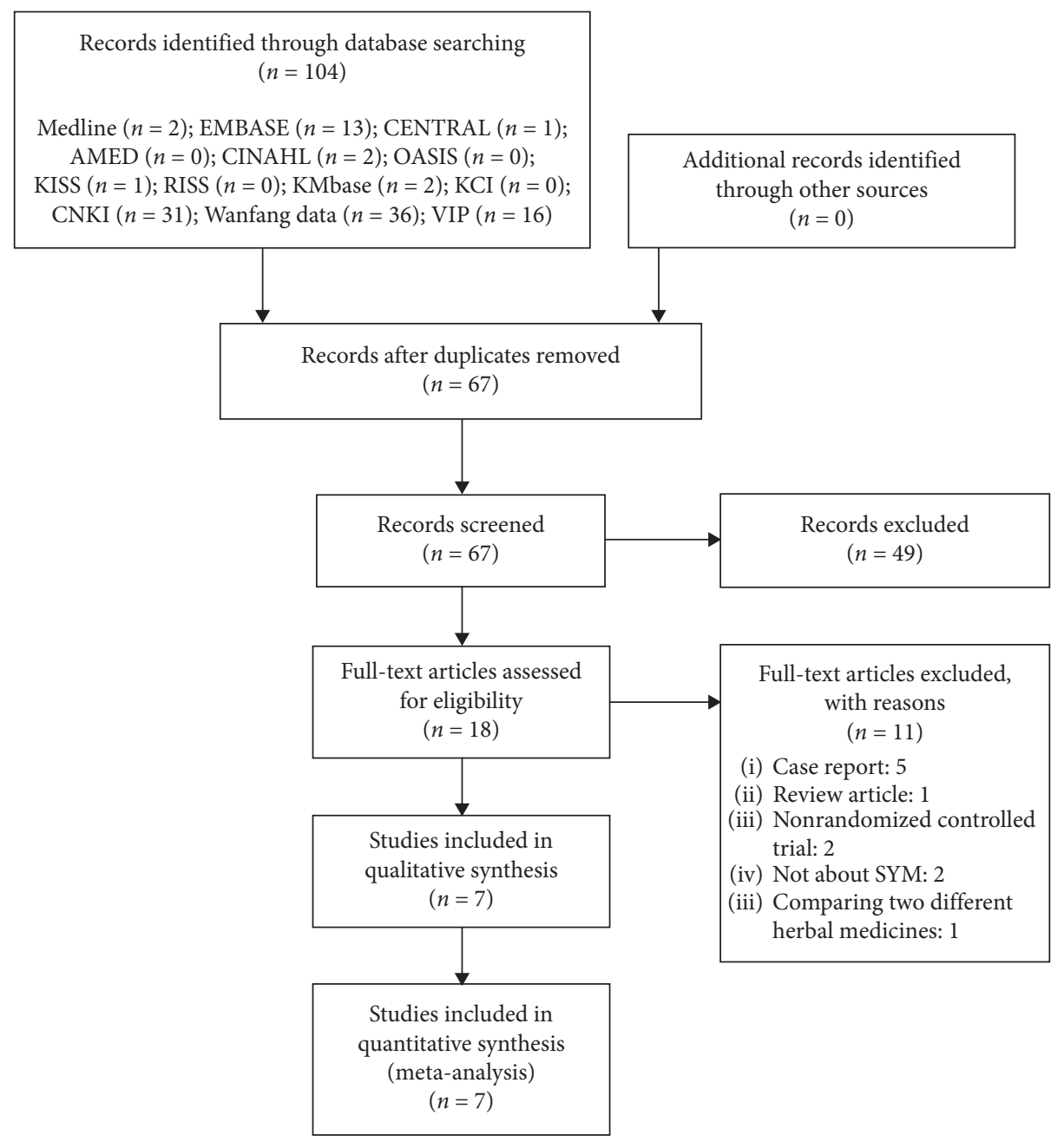

FIGURE 1: A PRISMA flow diagram of the literature screening and selection process. AMED, Allied and Complementary Medicine Database; CENTRAL, Cochrane Central Register of Controlled Trials; CINAHL, Cumulative Index to Nursing and Allied Health Literature; CNKI, China National Knowledge Infrastructure; KCI, Korea Citation Index; KISS, Korean studies Information Service System; KMbase, Korean Medical Database; OASIS, Oriental Medicine Advanced Searching Integrated System; RISS, Research Information Service System; SYM, Sihogayonggolmoryeo-tang.

including Deanxit (flupentixol and melitracen) were used in two studies $[30,35]$. Four studies included participants with hypertension [29, 31, 32, 34], two studies included participants with both hypertension and anxiety [30, 35], and one study included participants with both hypertension and depression [33]. One study targeted participants with isolated nocturnal hypertension [32]. Regarding the criteria for diagnosing hypertension, six studies were based on the Chinese Hypertension Prevention Guidelines [29-33, 35], and one study was based on the 2018 European guidelines for the prevention and treatment of hypertension [33]. The diagnosis criteria were not listed for one study [34]. Participants were recruited for four studies [29, 32, 33, 35] according to specific pattern identification: three [29, 32, 33] were for ascendant hyperactivity of liver yang, and the remaining study [35] was for yin deficiency with yang hyperactivity. Daytime SBP and DBP were evaluated in five studies [28-30, 32, 34], and nighttime SBP and DBP were measured in two studies $[28,31]$. As symptoms are related to hypertension, anxiety was assessed in two studies using the Zung self-rating anxiety score (SAS) [30] and Hamilton anxiety rating scale (HAMA) [35]. Depression was assessed in two studies using the Zung self-rating depression scale (SDS) [30] and patient health questionnaire-9 (PHQ-9) [33], respectively. TER calculated based on BP was evaluated in three studies [29, 32, 34], and TER calculated based on the traditional Chinese medicine (TCM) syndrome score was evaluated in four studies [29, 32, 33, 35]. The incidence of adverse events was reported in three studies [29, 33, 35], and quality of life was assessed in one study [29] using Du's hypertension quality of life scale (Table 1). Two articles $[30,33]$ reported that the studies had been approved by an institutional review board, and three articles [29, 30, 33] reported that the researchers had received consent from the participants.

Regarding the dosage form, a decoction was used in six studies [29-34], and granules were used in the remaining study [35]. At least one of the original components of SYM, 


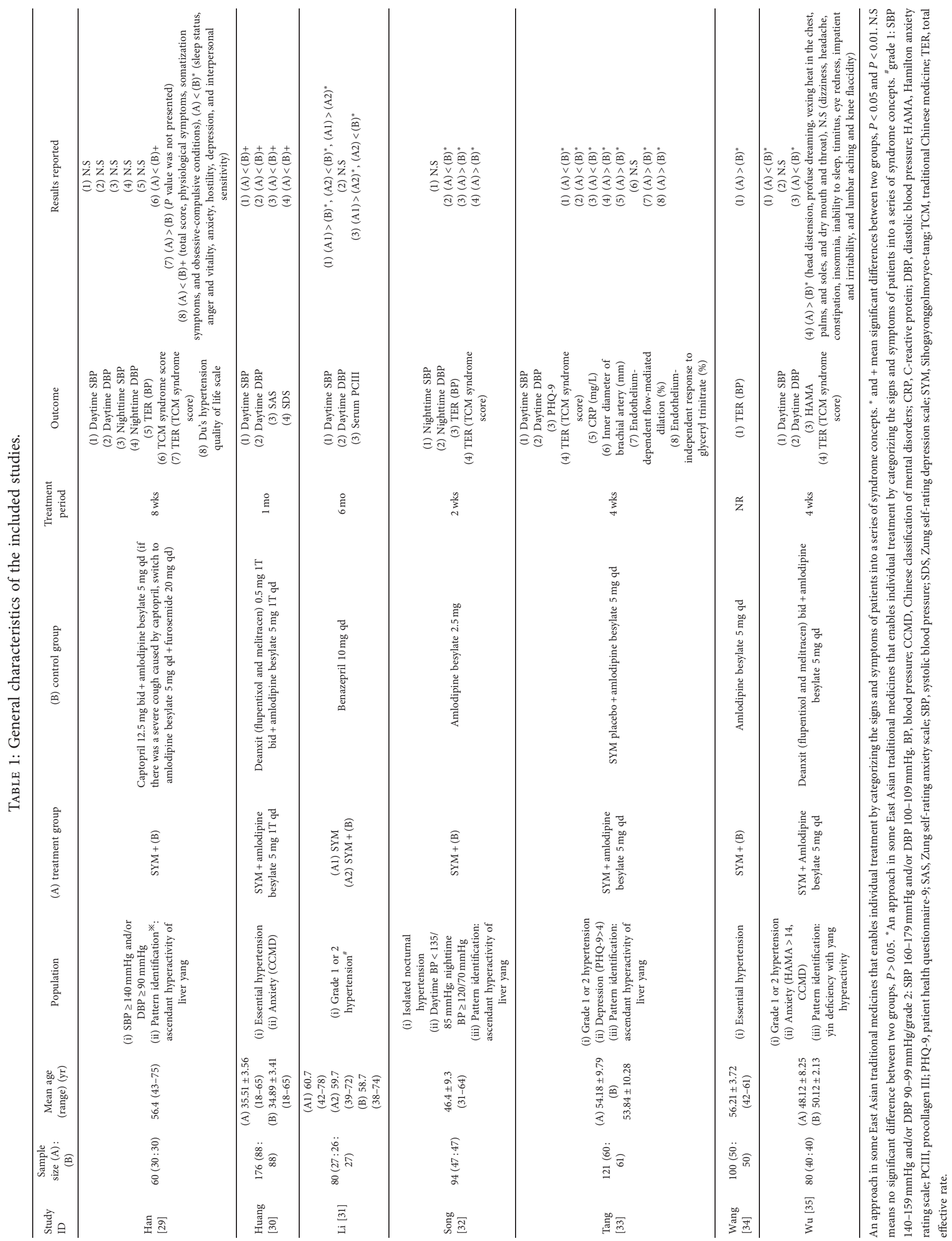


Bupleuri Radix, Fossilia Ossis Mastodi, Ostreae Testa, Scutellariae Radix, Pinelliae Tuber, Poria Sclerotium, and Cinnamomi Ramulus, was used in all studies (each 100\%). Additionally, Rhei Radix et Rhizoma was used in six studies (85.7\%) [29-31, 33-35], followed by Ginseng Radix or Codonopsis Pilosulae Radix [29-31, 33, 35] and Zingiberis Rhizoma Recens [29, 30, 33-35] in five studies (71.4\%) each. The administration duration varied from 2 weeks to 7 months, with 4 weeks being the most (Table 2).

3.3. Risk of Bias Assessment. For the random sequence generation domain, four studies [30, 32, 33, 35] were evaluated as having a low risk of bias because they used a random number table, and the remaining three studies $[29,31,34]$ were evaluated as having an unclear risk of bias because the relevant information was not provided. None of the articles provided information on allocation concealment and the blinding of outcome assessment; hence, the risk of bias was assessed as unclear. For the blinding of participants and personnel, one study [33] using placebo as a control was judged to have a low risk of bias, and the remaining studies [29-32, 34, 35] without any related information were judged to have a high risk of performance bias given the nature of the interventions used. For the incomplete outcome data domain, one study [30] was determined to have an unclear risk of attrition bias because the related information was not provided, and two studies $[33,35]$ were judged to have a high risk of bias due to analysis of the results using a per-protocol analysis method. One study [34] that reported only the TER calculated based on BP without reporting the raw data was evaluated to have a high risk of reporting bias. All studies were assessed to have a low risk of bias in other bias domains because they had demographic and clinical homogeneity at the baseline between the treatment and control groups (Figure 2).

\subsection{Efficacy}

3.4.1. SYM versus Placebo. Compared with placebo, SYM resulted in significantly lower daytime SBP and DBP (SBP: one study [33], $\mathrm{MD}-8.84 \mathrm{mmHg}, 95 \% \mathrm{CI}-15.36$ to -2.32 ; and DBP: one study [33], MD -9.11 mmHg, 95\% CI - 12.03 to -6.19). Additionally, depression measured with PHQ-9 was significantly lower in the SYM group (one study [33], MD -3.52 , 95\% CI -4.40 to -2.64 ). TER based on the TCM syndrome score after treatment was significantly higher in the SYM group than in the placebo group (one study [33], RR $1.21,95 \%$ CI 1.01 to 1.45 ) (Table 3 ).

3.4.2. SYM versus Active Controls. The SYM group showed significantly lower daytime SBP and DBP compared with the active control group (SBP: three studies [30, 31, 35], MD $-2.54 \mathrm{mmHg}, 95 \% \mathrm{CI}-4.18$ to $-0.89, \mathrm{I}^{2}=83 \%$; and DBP: three studies $[30,31,35], \mathrm{MD}-2.67,95 \% \mathrm{CI}-3.73$ to -1.60 , $\left.I^{2}=56 \%\right)$. However, subgroup analysis according to the type of active controls used to resolve considerable heterogeneity showed that daytime SBP was higher with SYM than with

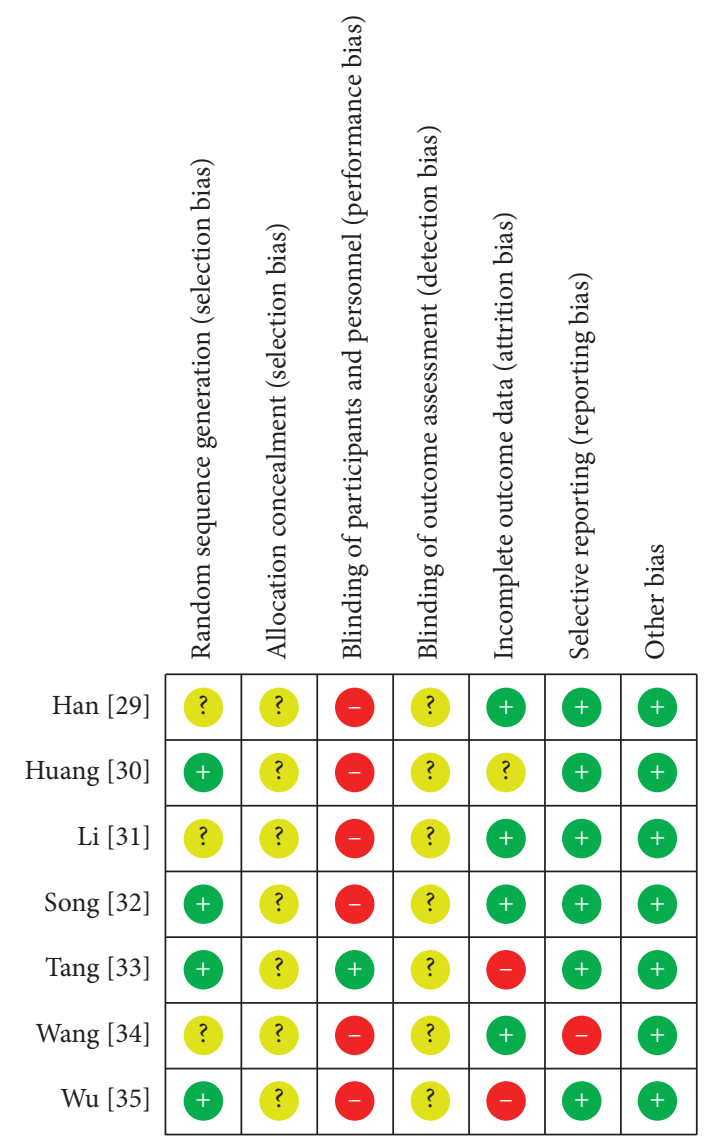

FIgURE 2: Risk of bias summary for all included studies. Low, unclear, and high risk, respectively, are represented with the following symbols: “+," "?," and “-."”

antihypertensives (one study [31]; MD $5.00 \mathrm{mmHg}$, 95\% CI 0.16 to 9.84 ), and daytime DBP showed no significant difference between the SYM and antihypertensives groups (one study [31]; MD $2.20 \mathrm{mmHg}, 95 \% \mathrm{CI}-2.73$ to 7.13 ). The degree of anxiety measured using SAS and HAMA showed no consistent results between the SYM and antidepressants groups (SAS: one study [30], MD $-10.58,95 \% \mathrm{CI}-11.57$ to -9.59; and HAMA: one study [35]; MD $-0.28,95 \%$ CI -0.95 to 0.39 ). The degree of depression measured with SDS showed significant results in favor of the SYM group (one study [30], MD $-10.94,95 \% \mathrm{CI}-12.17$ to -9.71 ) (Table 3).

3.4.3. SYM plus Active Controls versus Active Controls Alone. Antihypertensives were used as the active controls in all three studies included in this comparison. SYM plus antihypertensives resulted in significantly lower daytime SBP than antihypertensives alone (two studies [29, 31], MD $-3.22 \mathrm{mmHg}$, 95\% CI -6.15 to $\left.-0.29, I^{2}=0 \%\right)$. However, there were no significant differences between these two groups in daytime DBP, nighttime SBP, and nighttime DBP (daytime DBP: two studies [29, 31], $\mathrm{MD}-2.46 \mathrm{mmHg}, 95 \%$ CI -5.09 to $0.18, I^{2}=72 \%$; nighttime SBP: two studies $[29,32], \mathrm{MD}-1.79 \mathrm{mmHg}, 95 \% \mathrm{CI}-3.81$ to $0.23, I^{2}=0 \%$; and nighttime DBP: two studies [29, 32], $\mathrm{MD} 0.35 \mathrm{mmHg}$, $95 \% \mathrm{CI}-1.78$ to $\left.2.48, I^{2}=0 \%\right)$. The results were significant in 


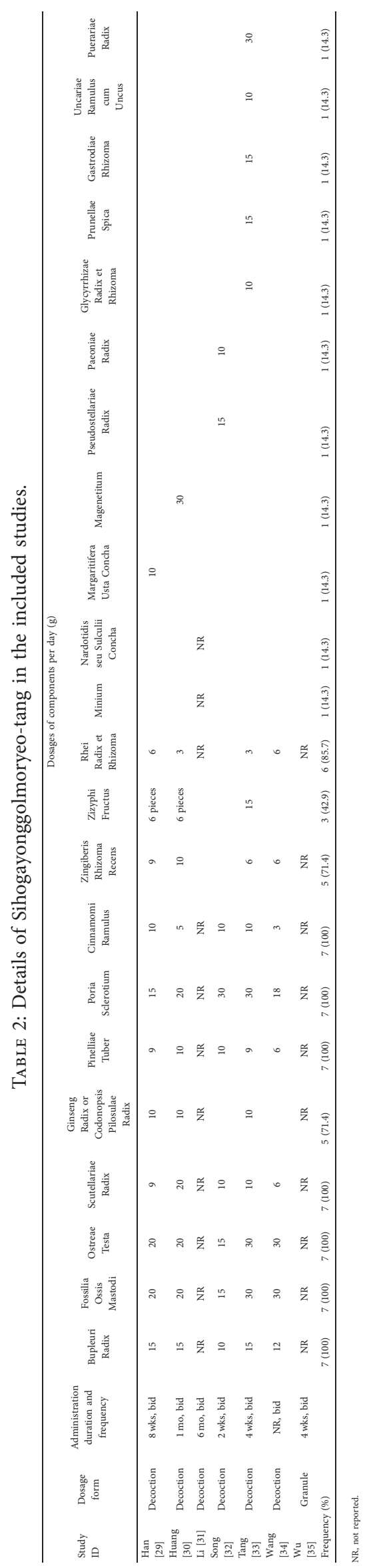


TABLe 3: Summary of findings.

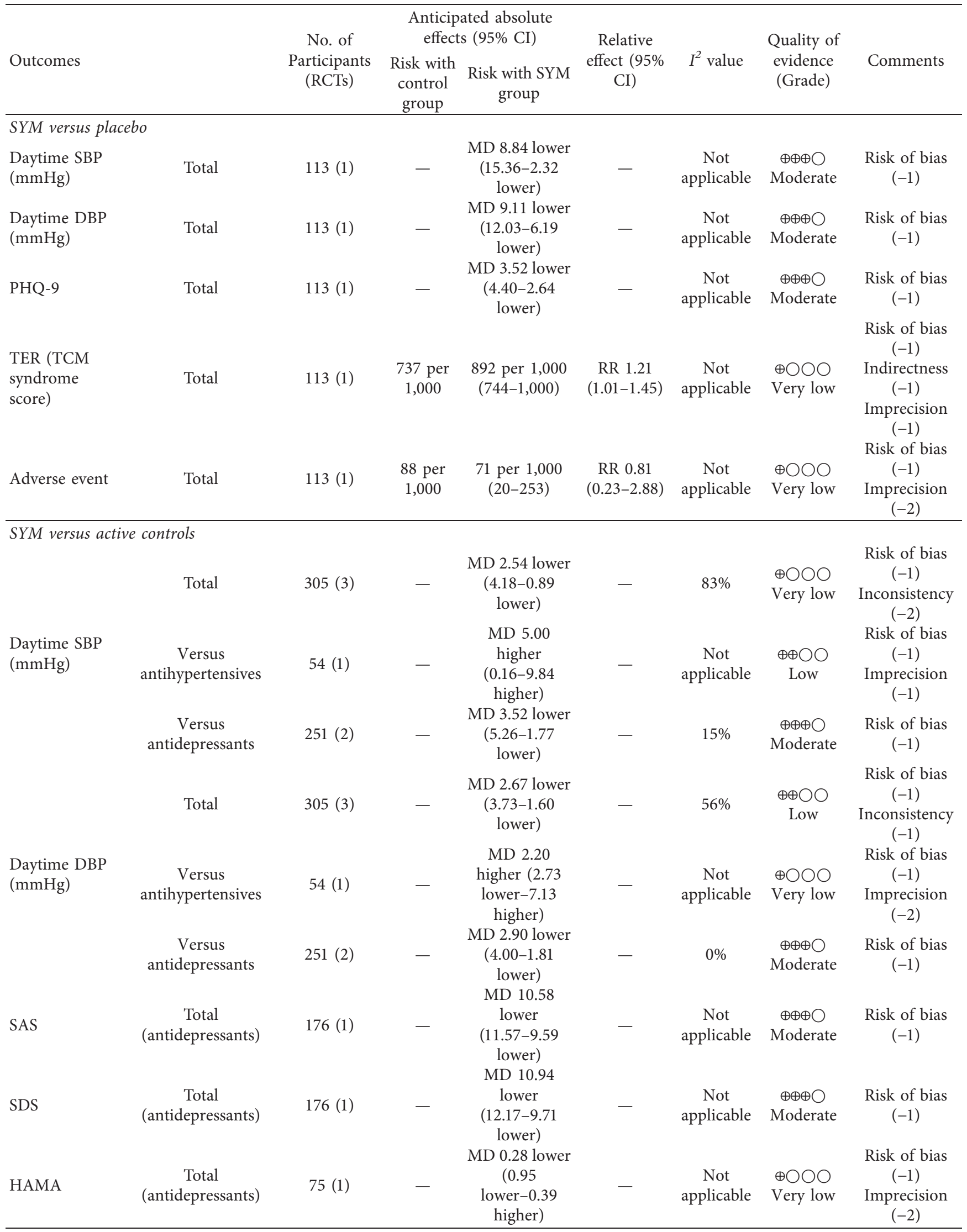


TABLE 3: Continued.

\begin{tabular}{|c|c|c|c|c|c|c|c|c|}
\hline \multirow{2}{*}{ Outcomes } & & \multirow{2}{*}{$\begin{array}{c}\text { No. of } \\
\text { Participants } \\
\text { (RCTs) }\end{array}$} & \multicolumn{2}{|c|}{$\begin{array}{l}\text { Anticipated absolute } \\
\text { effects }(95 \% \mathrm{CI})\end{array}$} & \multirow{2}{*}{$\begin{array}{c}\text { Relative } \\
\text { effect }(95 \% \\
\text { CI) }\end{array}$} & \multirow{2}{*}{$I^{2}$ value } & \multirow{2}{*}{$\begin{array}{l}\text { Quality of } \\
\text { evidence } \\
\text { (Grade) }\end{array}$} & \multirow[b]{2}{*}{ Comments } \\
\hline & & & $\begin{array}{c}\text { Risk with } \\
\text { control } \\
\text { group }\end{array}$ & $\begin{array}{l}\text { Risk with SYM } \\
\text { group }\end{array}$ & & & & \\
\hline Adverse event & $\begin{array}{c}\text { Total } \\
\text { (antidepressants) }\end{array}$ & $75(1)$ & $\begin{array}{l}0 \text { per } \\
1,000\end{array}$ & $\begin{array}{c}0 \text { per } 1,000 \\
(0-0)\end{array}$ & $\begin{array}{c}\text { Not } \\
\text { estimable }\end{array}$ & $\begin{array}{c}\text { Not } \\
\text { applicable }\end{array}$ & $\begin{array}{c}\oplus \oplus \bigcirc \bigcirc \\
\text { Low }\end{array}$ & $\begin{array}{c}\text { Risk of bias } \\
(-1) \\
\text { Imprecision } \\
(-1)\end{array}$ \\
\hline \multicolumn{9}{|c|}{ SYM plus active controls versus active controls } \\
\hline $\begin{array}{l}\text { Daytime SBP } \\
(\mathrm{mmHg})\end{array}$ & $\begin{array}{c}\text { Total } \\
\text { (antihypertensives) }\end{array}$ & $113(2)$ & - & $\begin{array}{c}\text { MD } 3.22 \text { lower } \\
(6.15-0.29 \\
\text { lower })\end{array}$ & - & $0 \%$ & $\begin{array}{l}\oplus \oplus \oplus \bigcirc \\
\text { Moderate }\end{array}$ & $\begin{array}{l}\text { Risk of bias } \\
\quad(-1)\end{array}$ \\
\hline $\begin{array}{l}\text { Daytime DBP } \\
(\mathrm{mmHg})\end{array}$ & $\begin{array}{c}\text { Total } \\
\text { (antihypertensives) }\end{array}$ & $113(2)$ & - & $\begin{array}{c}\text { MD } 2.46 \text { lower } \\
(5.09 \\
\text { lower- } 0.18 \\
\text { higher })\end{array}$ & - & $72 \%$ & $\begin{array}{l}\oplus \bigcirc \bigcirc \bigcirc \\
\text { Very low }\end{array}$ & $\begin{array}{c}\text { Risk of bias } \\
(-1) \\
\text { Inconsistency } \\
(-1) \\
\text { Imprecision } \\
(-1)\end{array}$ \\
\hline $\begin{array}{l}\text { Nighttime SBP } \\
(\mathrm{mmHg})\end{array}$ & $\begin{array}{c}\text { Total } \\
\text { (antihypertensives) }\end{array}$ & $154(2)$ & - & $\begin{array}{c}\text { MD } 1.79 \text { lower } \\
(3.81 \\
\text { lower- } 0.23 \\
\text { higher })\end{array}$ & - & $0 \%$ & $\begin{array}{c}\oplus \oplus \bigcirc \bigcirc \\
\text { Low }\end{array}$ & $\begin{array}{c}\text { Risk of bias } \\
(-1) \\
\text { Imprecision } \\
(-1)\end{array}$ \\
\hline $\begin{array}{l}\text { Nighttime DBP } \\
(\mathrm{mmHg})\end{array}$ & $\begin{array}{c}\text { Total } \\
\text { (antihypertensives) }\end{array}$ & $154(2)$ & - & $\begin{array}{c}\text { MD } 0.35 \\
\text { higher }(1.78 \\
\text { lower- } 2.48 \\
\text { higher })\end{array}$ & - & $0 \%$ & $\begin{array}{c}\oplus \oplus \bigcirc \bigcirc \\
\text { Low }\end{array}$ & $\begin{array}{c}\text { Risk of bias } \\
(-1) \\
\text { Imprecision } \\
(-1)\end{array}$ \\
\hline TER (BP) & $\begin{array}{c}\text { Total } \\
\text { (antihypertensives) }\end{array}$ & $254(3)$ & $\begin{array}{c}740 \text { per } \\
1,000\end{array}$ & $\begin{array}{c}903 \text { per } 1,000 \\
(807-1,000)\end{array}$ & $\begin{array}{l}\text { RR } 1.22 \\
(1.09 \text { to } \\
1.37)\end{array}$ & $61 \%$ & $\begin{array}{l}\oplus \bigcirc \bigcirc \bigcirc \\
\text { Very low }\end{array}$ & $\begin{array}{c}\text { Risk of bias } \\
\quad(-1) \\
\text { Indirectness } \\
\quad(-1) \\
\text { Imprecision } \\
(-1)\end{array}$ \\
\hline $\begin{array}{l}\text { TER (TCM } \\
\text { syndrome) }\end{array}$ & $\begin{array}{c}\text { Total } \\
\text { (antihypertensives) }\end{array}$ & $154(2)$ & $\begin{array}{c}688 \text { per } \\
1,000\end{array}$ & $\begin{array}{c}881 \text { per } 1,000 \\
(743-1,000)\end{array}$ & $\begin{array}{c}\text { RR } 1.28 \\
(1.08 \text { to } \\
1.52)\end{array}$ & $0 \%$ & $\begin{array}{c}\oplus \oplus \bigcirc \bigcirc \\
\text { Low }\end{array}$ & $\begin{array}{c}\text { Risk of bias } \\
(-1) \\
\text { Indirectness } \\
(-1)\end{array}$ \\
\hline $\begin{array}{l}\text { Du's } \\
\text { hypertension } \\
\text { quality of life } \\
\text { scale }\end{array}$ & $\begin{array}{c}\text { Total } \\
\text { (antihypertensives) }\end{array}$ & $60(1)$ & - & $\begin{array}{c}\text { MD 9.85 lower } \\
(15.87-3.83 \\
\text { lower })\end{array}$ & - & $\begin{array}{c}\text { Not } \\
\text { applicable }\end{array}$ & $\begin{array}{c}\oplus \oplus \bigcirc \bigcirc \\
\text { Low }\end{array}$ & $\begin{array}{c}\text { Risk of bias } \\
(-1) \\
\text { Imprecision } \\
(-1)\end{array}$ \\
\hline Adverse event & $\begin{array}{c}\text { Total } \\
\text { (antihypertensives) }\end{array}$ & $60(1)$ & $\begin{array}{l}33 \text { per } \\
1,000\end{array}$ & $\begin{array}{l}11 \text { per } 1,000 \\
(0-262)\end{array}$ & $\begin{array}{c}\text { RR } 0.33 \\
(0.01 \text { to } \\
7.87)\end{array}$ & $\begin{array}{c}\text { Not } \\
\text { applicable }\end{array}$ & $\begin{array}{l}\oplus \bigcirc \bigcirc \bigcirc \\
\text { Very low }\end{array}$ & $\begin{array}{c}\text { Risk of bias } \\
(-1) \\
\text { Imprecision } \\
(-2)\end{array}$ \\
\hline
\end{tabular}

BP, blood pressure; CI, confidence interval; DBP, diastolic blood pressure; HAMA, Hamilton anxiety rating scale; MD, mean difference; PHQ-9, patient health questionnaire-9; RCT, randomized controlled trial' RR, risk ratio; SAS, Zung self-rating anxiety scale; SBP, systolic blood pressure; SDS, Zung selfrating depression scale; SYM, Sihogayonggolmoryeo-tang; TCM, traditional Chinese medicine; TER, total effective rate.

favor of the SYM group for TER calculated based on BP (three studies $[29,32,34]$, RR $1.22,95 \%$ CI 1.09 to 1.37 , $I^{2}=61 \%$ ) and the TCM syndrome score (two studies $[29,32]$, RR $1.28,95 \%$ CI 1.08 to $\left.1.52, I^{2}=0 \%\right)$. The quality of life measured based on Du's hypertension quality of life scale was significantly improved in the SYM plus antihypertensives group compared with the antihypertensives-alone group (one study [29], MD $-9.85,95 \% \mathrm{CI}-15.87$ to -3.83 ) (Table 3).
3.4.4. Other Results. In one study [31], serum procollagen III, a biomarker of myocardial fibrosis, was significantly lower in the SYM plus benazepril group compared with the SYM alone or benazepril alone group (all, $P<0.05$ ). Tang et al. [33] reported that $C$-reactive protein was significantly lower in the SYM group than in the placebo group $(P<0.05)$. In addition, they reported that endothelium-dependent vasodilation was significantly improved in the SYM group compared with the placebo group $(P<0.05)$ (Table 1$)$. 
3.5. Safety. Of the seven studies, only three studies (42.86\%) $[29,33,35]$ reported adverse events. Tang et al.[33] reported two cases of blushing and two cases of constipation in the SYM group, with one case of ankle edema and four cases of constipation in the placebo group (RR 0.81, 95\% CI $0.23-2.88$ ). Han [29] reported no adverse events in the SYM group and one case of dry cough in the SYM plus antihypertensives group (RR $0.33,95 \%$ CI $0.01-7.87$ ). Wu et al. [35] reported no adverse events in the SYM and antidepressants groups (Table 3).

3.6. Quality of Evidence. In the comparison of SYM with placebo, the quality of evidence for daytime SBP, daytime DBP, and PHQ-9 was graded as "Moderate." However, TER calculated based on the TCM syndrome score and adverse events was graded as "Very Low." In the comparison of SYM with active controls and SYM plus active controls with active controls alone, the quality of evidence was graded as "Very Low" to "Moderate" (Table 3). The main reason for the downgrade was the high risk of bias for the included RCTs and the imprecision of the results due to the small sample size and wide CIs. Additionally, when TER was used, the indirectness of the outcome measure also lowered the quality of evidence.

3.7. Publication Bias. Because the number of studies included in each meta-analysis was less than 10, the assessment of publication bias through a funnel plot was not conducted.

\section{Discussion}

The aim of this study was to present evidence regarding the effect and safety of SYM for the treatment of hypertension through a comprehensive and systematic search, and seven studies [29-35] were included in the analysis. According to the study results, SYM significantly lowered BP and depressive symptoms in hypertensive patients compared with placebo. However, only one study [33] with an unclear risk of bias was included in this comparison, and no definite evidence could be obtained. SYM significantly lowered daytime BP compared to active control with considerable heterogeneity. Subgroup analysis according to the type of active controls used to resolve significant statistical and clinical heterogeneity revealed a significant decrease in the $I^{2}$ value. Interestingly, daytime SBP was significantly higher in the SYM group than in the antihypertensives group, but daytime DBP was not significantly different between the two groups. However, compared to the antidepressants group, daytime SBP and DBP were significantly lower in the SYM group. There was no significant difference in the concurrent anxiety symptoms when comparing SYM and antidepressants, but depression symptoms decreased significantly in the SYM group compared with that in the antidepressants group. When SYM plus antihypertensives was compared with antihypertensives alone, daytime SBP was significantly lower. However, daytime DBP and nighttime SBP showed borderline significance, and nighttime DBP had no significant difference between the two groups. However, TER calculated based on BP and quality of life was significantly higher in the SYM plus antihypertensives group. SYM showed no difference in the incidence of adverse events compared to placebo or active control, and the reported adverse reactions were mild and disappeared spontaneously. Most studies included had an unclear risk of bias, and the quality of evidence for the main findings was generally low.

Although hypertension itself is not a life-threatening problem, it can increase the risk of major medical conditions including cardiovascular and cerebrovascular diseases [36]. In addition, hypertension is reported to be associated with anxiety, depression, and insomnia [37-39], and the prevalence of depression among hypertensive patients is known to be moderate, at about 27\% [37]. According to the study results, SYM significantly improved the biomarkers of cardiovascular disease including serum procollagen III and C-reactive protein. In addition, given the increased demand for natural products that can control BP [10], the results of this study suggest the potential of SYM as a treatment option especially for hypertension and concurrent depressive symptoms. Modern pharmacological studies of SYM have shown that this HM can exert antidepressant effects by preventing the collapse of the hypothalamopituitary-adrenal (HPA) axis, including dysfunction in the glucocorticoid negative feedback system [16]. Dysfunction in the HPA axis is associated with negative cardiovascular outcomes, including hypertension, and is speculated to link hypertension and psychological stress [40]. Therefore, SYM can be assumed to have a beneficial effect on both hypertension and depression by restoring stress-related dysfunction in the HPA axis. Interestingly, among the studies included in this review, all studies using pattern identification [29, 32, 34, 35] recruited patients with yang hyperactivity, a concept related to psychological stress [41]. These findings support our hypothesis that the therapeutic effects of SYM may be involved in psychological stress and the HPA axis as a mediator for treating high BP and depression. However, the underlying mechanism of SYM on these conditions needs to be further elucidated.

According to previous studies [14], the pattern identification categories for hypertension are fire syndrome, phlegm-fluid retention syndrome, and deficiency syndrome. Among them, fire syndrome can be classified as a syndrome related to the liver, heart, stomach, and intestine. Based on the included studies using pattern identification and our previous study [42], the treatment principles for SYM were aimed at calming the liver and suppressing liver yang hyperactivity. Therefore, it can be assumed that SYM is used by some researchers under the category of fire syndrome, which mainly includes liver qi or liver yang abnormalities. However, only four of the studies included in this review $[29,32,34,35]$ recruited hypertensive patients with specific patterns, and we could not assess the effects of SYM in hypertensive patients according to specific patterns. Therefore, further study is needed to confirm whether SYM is more effective in hypertensive patients with a certain pattern.

There are some limitations to consider when interpreting the results. First, though we could not assess publication bias 
because less than 10 studies were included in this review, all included studies were conducted in China, suggesting potential reporting bias. Second, the risk of bias in all included studies was generally unclear; thus, the planned sensitivity analysis to identify the robustness of the meta-analysis results could not be conducted. Third, the incidence of cardiovascular and cerebrovascular events is an important outcome in hypertensive patients; however, none of the included studies conducted follow-up assessment after SYM administration. Nevertheless, some observational studies have reported the potential impact of HM on these outcomes. For example, in a previous cohort study [43], it was reported that HM improved the overall survival rate of hypertensive patients with type 2 diabetes. Long-term clinical trials or large-scale cohort studies are needed to evaluate the effects of SYM on cardiovascular and cerebrovascular events or mortality in hypertensive patients. Finally, although fatal adverse events induced by SYM were not observed in the included studies or in our previous study [42], only three studies (42.86\%) [29, 33, 35] reported the incidence of adverse events, and therefore, the safety profile of SYM remains unclear. Especially, although there was no age limit in the inclusion criteria for our study, and none of the included studies targeted children or pregnant women. Children may be even more susceptible to the adverse effects of HM because of their immature metabolic enzyme systems and an inappropriate dose per body weight. HMs also have the potential to cause adverse pregnancy outcomes and affect embryonic and fetal development in pregnant women, similar to conventional medication [44]. In this review, we could not find any evidence for the safety of SYM administration in this population, and further studies on the safety of SYM administration in this vulnerable population are needed. Moreover, due to concerns about potential adverse herbdrug interactions [45], the safety of HM in the treatment of hypertension should still be validated in well-designed clinical trials.

However, to the best of our knowledge, this is the first systematic review that comprehensively evaluated the effectiveness and safety of SYM for treating hypertensive patients. In addition, we significantly limited the statistical heterogeneity through subgroup analysis. Additional highquality RCTs with placebo control and large sample sizes should be conducted to provide conclusive evidence on SYM for hypertensive patients. In particular, to generalize the results, relevant studies should be conducted in other East Asian countries besides China. In addition, there is a need for further experimental studies on the mechanism of SYM in the treatment of hypertension.

\section{Conclusions}

Current evidence suggests that SYM may have positive effects on hypertension and concurrent depressive symptoms, especially compared with placebo or antidepressants, without serious adverse events. However, due to the unclear risk of bias in the included studies and low quality of evidence for the key findings, additional high-quality and placebo-controlled RCTs are needed to draw a definite conclusion.

\section{Data Availability}

The data used to support the findings of this study are included within the article.

\section{Disclosure}

Boram Lee and Chan-Young Kwon are co-first authors.

\section{Conflicts of Interest}

The authors have no conflicts of interest to declare.

\section{Authors' Contributions}

Boram Lee and Chan-Young Kwon contributed equally to this work. This study was conceptualized by BL. The study search, screening, data extraction, and quality assessment were conducted by $\mathrm{BL}$, and CYK cross-checked it. The manuscript was drafted and revised by BL and CYK. All authors have read and approved the final manuscript.

\section{Acknowledgments}

This work was supported by the Korea Institute of Oriental Medicine (KSN2013210).

\section{Supplementary Materials}

Supplement 1. Search terms used in each database. Supplement 2. PRISMA 2009 checklists. (Supplementary Materials)

\section{References}

[1] WHO, Global Health Risks: Mortality and Burden of Disease Attributable to Selected Major Risks, World Health Organization, Geneva, Switerzland, 2009.

[2] S. S. Lim, T. Vos, A. D. Flaxman et al., "A comparative risk assessment of burden of disease and injury attributable to 67 risk factors and risk factor clusters in 21 regions, 1990-2010: a systematic analysis for the Global Burden of Disease Study 2010," Lancet (London, England), vol. 380, no. 9859, pp. 2224-2260, 2012.

[3] K. T. Mills, J. D. Bundy, T. N. Kelly et al., "Global disparities of hypertension prevalence and control," Circulation, vol. 134, no. 6, pp. 441-450, 2016.

[4] N. Ikeda, D. Sapienza, R. Guerrero et al., "Control of hypertension with medication: a comparative analysis of national surveys in 20 countries," Bulletin of the World Health Organization, vol. 92, no. 1, pp. 10-9c, 2014.

[5] R. M. Carey, S. Sakhuja, D. A. Calhoun, P. K. Whelton, and P. Muntner, "Prevalence of apparent treatment-resistant hypertension in the United States," Hypertension (Dallas, Texas), vol. 73, no. 2, pp. 424-431, 1979.

[6] C. Bardage and D. G. Isacson, "Self-reported side-effects of antihypertensive drugs: an epidemiological study on 
prevalence and impact on health-state utility," Blood Pressure, vol. 9, no. 6, pp. 328-334, 2000.

[7] M. Morgado, S. Rolo, A. F. Macedo, L. Pereira, and M. Castelo-Branco, "Predictors of uncontrolled hypertension and antihypertensive medication nonadherence," Journal of Cardiovascular Disease Research, vol. 1, no. 4, pp. 196-202, 2010.

[8] W. S. Aronow, "Lifestyle measures for treating hypertension," Archives of Medical Science, vol. 5, no. 5, pp. 1241-1243, 2017.

[9] J. J. Beunza, M. A. Martínez-González, S. Ebrahim et al., "Sedentary behaviors and the risk of incident hypertension: the SUN Cohort," American Journal of Hypertension, vol. 20, no. 11, pp. 1156-1162, 2007.

[10] J. Mbizo, A. Okafor, M. A. Sutton, B. Leyva, L. M. Stone, and O. Olaku, "Complementary and alternative medicine use among persons with multiple chronic conditions: results from the 2012 National Health Interview Survey," BMC Complementary and Alternative Medicine, vol. 18, no. 1, p. 281, 2018.

[11] R. Nahas, "Complementary and alternative medicine approaches to blood pressure reduction: an evidence-based review," Canadian family physician Medecin de famille canadien, vol. 54, no. 11, pp. 1529-1533, 2008.

[12] C.-h. Han, M. Kim, S.-Y. Cho et al., “Adjunctive herbal medicine treatment for patients with acute ischemic stroke: a systematic review and meta-analysis," Complementary Therapies in Clinical Practice, vol. 33, pp. 124-137, 2018.

[13] L. Yu, Y. Qin, Q. Wang et al., "The efficacy and safety of Chinese herbal medicine, Rhodiola formulation in treating ischemic heart disease: a systematic review and meta-analysis of randomized controlled trials," Complementary Therapies in Medicine, vol. 22, no. 4, pp. 814-825, 2014.

[14] J. Wang and X. Xiong, "Evidence-based Chinese medicine for hypertension," Evidence-Based Complementary and Alternative Medicine, vol. 2013, p. 97, 2013.

[15] K. Mizoguchi, R. Ikeda, H. Shoji et al., "Saikokaryukotsuboreito, a herbal medicine, prevents chronic stressinduced anxiety in rats: comparison with diazepam," Journal of Natural Medicines, vol. 63, no. 1, pp. 69-74, 2009.

[16] K. Mizoguchi, N. Sun, X.-L. Jin et al., "Saikokaryukotsuboreito, a herbal medicine, prevents chronic stressinduced dysfunction of glucocorticoid negative feedback system in rat brain," Pharmacology Biochemistry and Behavior, vol. 86, no. 1, pp. 55-61, 2007.

[17] K. Mizoguchi, M. Yuzurihara, A. Ishige, M. Aburada, and T. Tabira, "Saiko-ka-ryukotsu-borei-to, a herbal medicine, ameliorates chronic stress-induced depressive state in rotarod performance," Pharmacology Biochemistry and Behavior, vol. 75, no. 2, pp. 419-425, 2003.

[18] H. Iijima, A. Daikonya, S. Takamatsu et al., "Effects of the herbal medicine composition "Saiko-ka-ryukotsu-borei-To" on the function of endothelial progenitor cells in hypertensive rats," Phytomedicine: International Journal of Phytotherapy and Phytopharmacology, vol. 20, no. 3-4, pp. 196-201, 2013.

[19] H. Okano and C. Ohkubo, "Anti-pressor effect of a ChineseJapanese herbal medicine, saiko-ka-ryukotsu-borei-to on hemodynamics in rabbits," Vivo (Athens, Greece), vol. 13, no. 4, pp. 333-337, 1999.

[20] M. Wei, F. Shintani, S. Kanba et al., "Endothelium-dependent and-independent vasoactive actions of a Japanese kampo medicine, saiko-ka-ryukotsu-borei-to," Biomedicine \& Pharmacotherapy, vol. 51, no. 1, pp. 38-43, 1997.

[21] D. Moher, A. Liberati, J. Tetzlaff, and D. G. Altman, "Preferred reporting items for systematic reviews and meta-analyses: the PRISMA statement," PLoS Medicine, vol. 6, no. 7, 2009.
[22] B. Lee, C.-Y. Kwon, and G. T. Chang, "Acupoint herbal patching for children with recurrent respiratory tract infection: a systematic review and meta-analysis," Complementary Therapies in Clinical Practice, vol. 40, p. 101209, 2020.

[23] S. S. K. Durairajan, A. Iyaswamy, S. G. Shetty et al., "A modified formulation of Huanglian-Jie-Du-Tang reduces memory impairments and beta-amyloid plaques in a triple transgenic mouse model of Alzheimer's disease," Scientific Reports, vol. 7, no. 1, p. 6238, 2017.

[24] T. Yamada, T. Wajima, H. Nakaminami, K. Kobayashi, H. Ikoshi, and N. Noguchi, "The modified Gingyo-san, a Chinese herbal medicine, has direct antibacterial effects against respiratory pathogens," BMC Complementary and Alternative Medicine, vol. 16, no. 1, p. 463, 2016.

[25] J. P. T. Higgins, D. G. Altman, P. C. Gotzsche et al., "The Cochrane Collaboration's tool for assessing risk of bias in randomised trials," Bmj, vol. 343, no. 2, p. d5928, 2011.

[26] H. Balshem, M. Helfand, H. J. Schünemann et al., "GRADE guidelines: 3. Rating the quality of evidence," Journal of Clinical Epidemiology, vol. 64, no. 4, pp. 401-406, 2011.

[27] G. C. de Souza, A. C. Matias Pereira, M. D. Viana et al., "Acmella oleracea (L) R. K. Jansen reproductive toxicity in zebrafish: an in vivo and in silico assessment," Evidence-Based Complementary and Alternative Medicine, vol. 2019, 2019.

[28] M. Borenstein, L. V. Hedges, J. P. T. Higgins, and H. R. Rothstein, "A basic introduction to fixed-effect and random-effects models for meta-analysis," Research Synthesis Methods, vol. 1, no. 2, pp. 97-111, 2010.

[29] M. Han, The Therapeut Effect of Longgu Muli Decoction on Quality of Life of Patients with Hypertension, Shandong University of Traditional Chinese Medicine, Berlin, Germany, 2013.

[30] W. M. Huang, "Effect comparison of Amlodipine Besylate combined with Chaihu plusing Longgu Oyster decoction or Flupentixol and Melitracen treating primary hypertension complicated with anxiety," China Modern Medicine, vol. 24, no. 14, pp. 122-124, 2017.

[31] X. Q. Li and Y. K. Li, "Effect of chaihu jia longgu muli decoction on serum PCIII in patients with hypertension," Seek Medical And Ask The Medicine, vol. 10, no. 3, p. 491, 2012.

[32] X. X. Song, C. H. Yan, X. T. Zhang, and A. M. Liu, "Clinical observation of Caihulonggumuli decoction in adjuvant treatment of isolated nocturnal hypertension with hyperactivity of liver yang," Hubei Journal of TCM, vol. 38, no. 5, pp. 7-8, 2016.

[33] Y. L. Tang, J. M. Wang, T. Y. Zhuang, C. Yang, and J. P. Fu, "Efficacy and safety of modified Chaihu Jia Longgu Muli tang in treating mild to moderate essential hypertension complicated with depression and liver-yang hyperactivity syndrome," Chinese Journal of Experimental Traditional Medical Formulae, vol. 38, pp. 1-7, 2020.

[34] X. F. Wang, "Clinical observation of Chaihu Jia Longgu Muli decoction in treating essential hypertension," Journal of Medical Aesthetics and Cosmetology, vol. 28, no. 23, p. 65, 2019.

[35] X. F. Wu, X. Z. Xie, G. L. Xu, and J. Y. Wang, "Clinical observation of Chaihu Jia Longgu Muli decoction in the treatment of essential hypertension with anxiety," World Journal of Integrated Traditional and Western Medicine, vol. 11, no. 11, pp. 1497-1499, 2016.

[36] B. C. Bansal, A. K. Agarwal, and B. B. Rewari, "Hypertension and cerebrovascular disease," Journal of the Indian Medical Association, vol. 97, no. 6, pp. 226-232, 1999. 
[37] Z. Li, Y. Li, L. Chen, P. Chen, and Y. Hu, "Prevalence of depression in patients with hypertension," Medicine, vol. 94, no. 31, p. e1317, 2015.

[38] Y. Pan, W. Cai, Q. Cheng, W. Dong, T. An, and J. Yan, "Association between anxiety and hypertension: a systematic review and meta-analysis of epidemiological studies," Neuropsychiatric Disease and Treatment, vol. 11, pp. 1121-1130, 2015.

[39] A. N. Vgontzas, D. Liao, E. O. Bixler, G. P. Chrousos, and A. Vela-Bueno, "Insomnia with objective short sleep duration is associated with a high risk for hypertension," Sleep, vol. 32, no. 4, pp. 491-497, 2009.

[40] N. Burford, N. Webster, and D. Cruz-Topete, "Hypothalamicpituitary-adrenal Axis modulation of glucocorticoids in the cardiovascular system," International Journal of Molecular Sciences, vol. 18, no. 10, p. 2150, 2017.

[41] Q. Yan, "Neuroimmune imbalances and yin-yang dynamics in stress, anxiety, and depression," Methods in Molecular Biology, vol. 1781, pp. 77-85, 2018.

[42] C. Y. Kwon, B. Lee, S. Y. Chung et al., "Efficacy and safety of Sihogayonggolmoryeo-tang (Saikokaryukotsuboreito, Chai$\mathrm{Hu}$-Jia-Long-Gu-Mu-Li-Tang) for post-stroke depression: a systematic review and meta-analysis," Scientific Reports, vol. 9, no. 1, p. 146, 2019.

[43] Y. J. Lin, T. J. Ho, Y. C. Yeh et al., "Chinese herbal medicine treatment improves the overall survival rate of individuals with hypertension among type 2 diabetes patients and modulates in vitro smooth muscle cell contractility," PloS One, vol. 10, no. 12, 2015.

[44] C. C. Wang, L. Li, C. B. San Lau, P. C. Leung, and K. P. Fung, "Pregnancy outcomes, embryonic and fetal development in maternal exposure to Chinese medicines," Birth Defects Research Part C: Embryo Today: Reviews, vol. 99, no. 4, pp. 275-291, 2013.

[45] A. Tachjian, V. Maria, and A. Jahangir, "Use of herbal products and potential interactions in patients with cardiovascular diseases," Journal of the American College of Cardiology, vol. 55, no. 6, pp. 515-525, 2010. 